\title{
PHYSICS OF CHARGED COSMIC RAYS WITH THE AMS EXPERIMENT
}

\author{
J.P. VIALLE \\ LAPP, IN2P3-CNRS, Chemin de Bellevue, BP110, \\ F-74941, Annecy-le-Vieux
}

\begin{abstract}
The AMS experiment aims at searching for primordial antimatter, non-baryonic dark matter, and measuring with high statistics and high accuracy the electrically charged cosmic ray particles and light nuclei in the extraterrestrial space beyond the atmosphere. AMS is the first magnetic spectrometer which will be flown in space. It will be installed for 3 years on the international space station (ISS) in 2003. A test flight with the space shuttle DISCOVERY took place in June 1998 with a first detector and gave many results: best limit on the existence of antinuclei, fluxes of protons, leptons, and helium nuclei above the geomagnetic threshold, existence of a secondary flux below the geomagnetic threshold. These results are described below. The physics goal and perspectives for AMS on the space station with an improved detector are described as well
\end{abstract}

Talk given at the IVth Rencontres du Vietnam, July 19-25, 2000, Hanoi, Vietnam

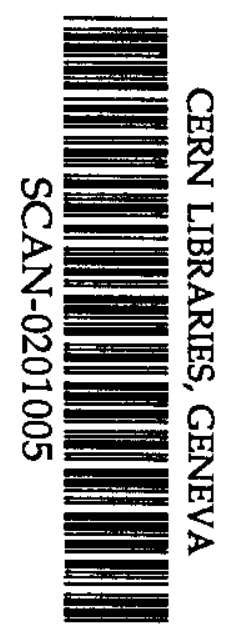




\title{
PHYSICS OF CHARGED COSMIC RAYS WITH THE AMS EXPERIMENT
}

\author{
J.P. VIALLE ${ }^{a}$ \\ LAPP-IN2P3/CNRS/University of Savoie \\ B.P. 110, F-74941 Annecy-le-Vieux Cedex \\ AMS Collaboration
}

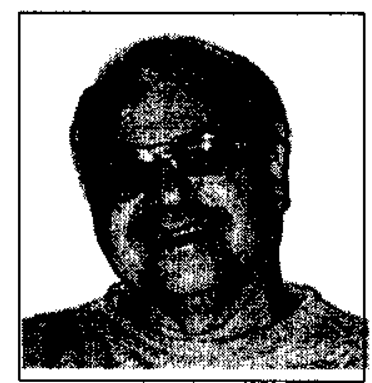

\begin{abstract}
The AMS experiment aims at searching for primordial antimatter, non-baryonic dark matter, and measuring with high statistics and high accuracy the electrically charged cosmic ray particles and light nuclei in the extraterrestrial space beyond the atmosphere. AMS is the first magnetic spectrometer which will be flown in space. It will be installed for 3 years on the international space station (ISS) in 2003. A test flight with the space shuttle DISCOVERY took place in June 1998 with a first detector and gave many results: best limit on the existence of antinuclei, fluxes of protons, leptons, and helium nuclei above the geomagnetic threshold, existence of a secondary flux below the geomagnetic threshold. These results are described below. The physics goal and perspectives for AMS on the space station with an improved detector are described as well
\end{abstract}

\section{Introduction}

Gamma rays propagation in the intergalactic and interstellar medium is not affected by magnetic fields, and thus it is possible to point at sources of gamma rays and to identify it.

On the contrary, the information of direction of the sources of charged particles is washed out by the propagation in the galactic and intergalactic magnetic field, and in the earth magnetic field as well. Near the earth, the fluxes of charged cosmic rays are disturbed by the solar activity which produces the solar wind, a plasma made mainly of protons and electrons which acts like a shielding for the earth. Despite these effects, charged cosmic rays contain physics information on the dynamics of galaxies (cosmic wind, confinement, origin of charged cosmic rays) and on primordial universe that cannnot be found elsewhere.

Due to the difficulty to build a magnetic spectrometer and operate it in space, AMS will be the first magnetic spectrometer in space for a long duration, 3 to 5 years. The main goal of the

\footnotetext{
${ }^{a}$ e-mail: vialle@lapp.in2p3.fr
} 
experiment is the search for primordial antimatter, for non-baryonic dark matter, and the high precision and high statistics measurement of electrically charged cosmic ray particles and light nuclei in a very wide range of energy, from a few hundred $\mathrm{MeV}$ up to a few $\mathrm{TeV}$ in momentum.

A technical flight of eleven days on board of the space shuttle DISCOVERY took place in june 1998 to test the detector behaviour under actual space flight conditions, to gather data on the background sources, and to study the operating parameters of the experiment.

\section{The physics of AMS}

\subsection{Primordial antimatter}

It was demonstrated by A. Zacharov that to generate an asymmetric universe made of matter only, starting from a symmetric universe, i.e. an universe in which there is the same amount of matter and of antimatter, as it is in the big-bang theory at the very beginning of the universe, 4 conditions must be fulfilled : i) Violation of the baryonic number ii) Violation of the charge conjugation $\mathrm{C}$ iii) large $\mathrm{CP}$ violation iv) Baryogenesis out of the thermal equilibrium. These conditions are not supported by experimental data. Baryon non-conservation and large levels of $\mathrm{CP}$-violation have not been observed up to now. On the other hand, the absence of observation of gamma ray peaks from matter-antimatter annihilation excludes the presence of large quantity of antimatter within a distance of the order of $10 \mathrm{Mpc}$ from the earth.

A clear signature of the existence of islands of antimatter in the universe would be the observation of antinuclei of Helium or Carbon, which can only be produced in the furnace of a star of antimatter, and be ejected in the outer space in a supernova explosion. Balloon borne experiments have searched for such antinuclei for more than 20 years with negative results ${ }^{2,3,4}$. AMS is designed to reach a sensitivity of $10^{-9}$ on the ratio antihelium/helium, better by 3 order of magnitude than the existing experiments.

\subsection{Dark matter}

From the motion of the spiral arms of galaxies and of the cluster of galaxies in pareticular it can be deduced that about $90 \%$ of the mass of the universe is non-radiating, and therefore is invisible to classical astrophysics experiments. The nature of this so-called dark matter is not yet known, but from the experimental data $a^{5}$ it is proven that most of the dark matter is of non baryonic origin. These objects are called WIMP's. In supersymmetric theories, a good candidate for WIMP's is the neutralino $\chi$, the lightest supersymmetric particle, which could be observed through the particles produced in its annihilation like antiprotons, positrons, and gammas ${ }^{6}$. AMS will be able to measure accurately the fluxes of antiprotons and positrons from $.5 \mathrm{GeV} / \mathrm{c}$ to $1 \mathrm{TeV} / \mathrm{c}$.

\subsection{Ordinary matter}

A lot of information on the mechanisms of galaxies is buried in the abundance and in the spectra of charged particles and nuclei. The spectra of charged cosmic rays at high energy falls off according to a power law, and the slope contains information about the origin of such cosmic rays, either primary or secondary. Unexpected phenomena could show up as a distortion of such spectra. For light nuclei, the isotopic content, gives information on the confinement of cosmic rays in the galaxy. The unstable isotopes act like a clock which gives the time spent in the galaxy by the nuclei between their production and the measurement. This is for instance the case for the $\mathrm{Be}^{9}$ and $\mathrm{Be}^{10}$, of which the ratio of abundance gives a direct measurement of the confinement time. AMS will be able to measure accurately all the fluxes of light nuclei on a kinetic energy range from few tens of $\mathrm{MeV} / \mathrm{Nucleon}$ up to around $15 \mathrm{GeV} / \mathrm{N}$, and the 


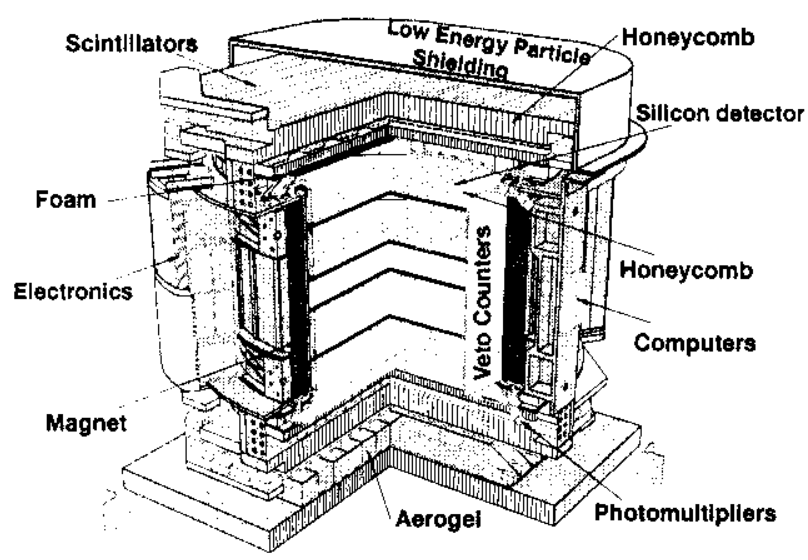

Figure 1: The AMS01 detector

particle spectra up to about $1 \mathrm{TeV}$, providing invaluable informations for the understanding of the mechanisms of galaxies.

\section{The AMS01 Spectrometer}

For the AMS01 precursor flight, AMS consisted mainly of a permanent magnet, a silicon tracker, time of flight hodoscopes, anticoincidence counters, and an aerogel threshold Cerenkov counter. The acceptance was about $0.3 \mathrm{~m}^{2} \mathrm{sr}$. The permanent magnet was made of 1.9 tons of Nd-Fe-B in the shape of a cylindrical shell of inner diameter $1115 \mathrm{~mm}$, providing a dipole field of 0.14 Tesla in the $\mathrm{x}$ direction perpendicular to the cylinder axis, and an analysis power $\mathrm{BL}^{2}$, parallel to the magnet axis $\mathrm{z}$ of $0.14 \mathrm{Tm}^{2}$. The trajectory of particles traversing the magnet bore was measured by six planes of double sided silicon microstrip detectors put inside the magnet perpendicular to the axis, the outer layers being just outside the magnet cylinder, above and below. From the deflection the rigidity $\mathrm{R}=\mathrm{pc} /|Z| \mathrm{e}(\mathrm{GV})$ was measured. The tracker had an accuracy of about 10 microns in the bending plane and 30 microns in the other one. It provided also a determination of the charge $|Z|$ through multiple measurements of energy loss. The total amount of material of the tracker as seen by a particle parallel to the axis of the magnet was only $3 \%$ of radiation length, in order to minimize interactions and multiple scattering.

The particle velocity and direction of motion were measured by a time-of-flight (TOF) system made of four layers of scintillation counters, 2 above and 2 below the magnet. The typical accuracy on the time measurement was $120 \mathrm{psec}$ for particles of unit charge, and $105 \mathrm{psec}$ for charge $|Z|=2$, allowing to separate electrons from protons up to $1.5 \mathrm{GeV} / \mathrm{c}$. The pulse height information from each plane provided additional determination of the charge $|Z|$. The velocity measurement was complemented by a threshold Cerenkov counter ${ }^{3}$ with an aerogel radiator of refractive index $n=1.035$, giving a separation between electrons and protons up to $3.7 \mathrm{GeV} / \mathrm{c}$. A layer of anticoincidence scintillation counters lined the inner surface of the magnet to get rid of the background caused by particles passing through or interacting in the magnet walls and support structure. Finally, the detector was also shielded from low energy (a few MeV) particles by thin carbon fiber walls. The basic trigger was made of the coincidence of signals in 3 out of 4 of the TOF planes, with a veto on a signal in the anticoincidence counters. During the construction, the detector components went successfully through extensive space qualification tests (acceleration, vibration, thermal vacuum, electromagnetic interference, and radiation).

During the flight, the detector performances as well as the temperature and the magnetic field were monitored continuously, and the alignment of the tracker was also continuously monitored with an infrared laser system. After the flight, the detector was extensevely checked at two accelerators: At GSI Darmstadt with Helium and Carbon beams from 1.0 to $5.6 \mathrm{GV}$ in rigidity 
at 600 incident angles and locations for a total of $10^{7}$ events, and at CERN-PS with protons of 2 to $14 \mathrm{GeV}$ at 1200 incident angles and locations for a total of $10^{8}$ events. This ensured that the behaviour of the detector and its performances on momentum measurement, mass separation and charge separation were thoroughly understood.For instance, from the beam of $10 \pm 0.1 \mathrm{GeV}$ protons, the tracks reconstructed gave a mean value of $10.04 \mathrm{GeV}$ with a width of $0.92 \mathrm{GeV}$, showing that there is no bias in the reconstruction.

\section{AMS01 on flight STS-91}

During the flight STS-91 the orbit was inclined by $51.6^{\circ}$ on the geographic equator, at an altitude varying between $320 \mathrm{kms}$ and $390 \mathrm{kms}$. More than 100 millions triggers in total were gathered during three period : a) 25 hours before docking on the MIR station, during which the AMS z-axis was kept pointing within $45^{\circ}$ of the zenith. b) 4 days while docked to MIR. The AMS z-axis pointing varied between $40^{\circ}$ and $145^{\circ}$ of the zenith. c) After MIR undocking. Within $1^{\circ}$, the pointing was kept at $0^{\circ}, 20^{\circ}$, and $45^{\circ}$ of the zenith for 19,25 , and 20 hours respectively. Eventually, 4 hours of data tacking were done before descending, with the shuttle in reversed position and the AMS z-axis pointing towards the nadir, in order to have measurement of the fluxes of upward moving particles.

The fluxes of cosmic rays from outer space is affected by the earth magnetic field which bends their trajectories back to space when the energy is below a threshold that depends on the impinging angle and the geomagnetic latitude of the particle. The earth magnetic field is in first approximation a dipole inclined of about $11^{\circ}$ with respect to the earth rotation axis, and the south magnetic pole near the north geographic pole. The dipole axis is shifted by about 400 $\mathrm{kms}$ from the center of the earth. Near the magnetic pole the kinetic energy threshold is as low as $10 \mathrm{MeV}$ while at the magnetic equator it raises beyond $10 \mathrm{GeV}$. In the South Atlantic the magnetic field is much weaker (i.e. the so-called SAA or South Atlantic Anomaly) which allows a high flux of low energy particles to reach the Earth. Data taken while passing through or near the SAA were excluded from all the analysis.

\section{Search for antihelium}

For this $\operatorname{search}^{10}$, the sample was restricted to data taken with the shuttle attitude such that the z-axis of AMS was pointing toward the zenith within an angle of 45 degrees. Events were selected after full reconstruction (momentum, sign, velocity, direction upward or downward, energy loss in the tracker and in the TOF estimated by truncated mean) and were required to give hits in at least 4 tracker planes out of 6 , and to have an energy loss in tracker and in TOF compatible with $\mathrm{Z}=2$ charge. The latter criteria rejected the background of electrons with wrongly measured charge, since the combined charge confusion probability was found to be less than $10^{-7}$ (fig 2). The possibility of misidentifying the direction upward or downward of the cosmic ray was found to be negligible from a control sample. To get rid of large angle nuclear scattering on one of the tracker plane, it was also requested that the parameters of the track reconstructed with the first 3 hits, the last 3 hits, and using all the hits in the tracker be compatible. To remove events with colinear delta rays which could disturb the reconstruction a last cut was applied on events with an excess of energy deposited within $\pm 5 \mathrm{~mm}$ of the track. Finally a probability function was constructed from the measurement of velocity, rigidity, and energy loss to to check the compatibility of the track with the passage of an helium or antihelium nuclei with $A=3$ or 4 . While this last cut removed the 4 last remaining antihelium candidates, the Helium sample was very little affected. A total of $2.8 \cdot 10^{6} \mathrm{He}$ was obtained in a rigidity range of up to $140 \mathrm{GV}$ while there was no antihelium at any rigidity(fig 2). The limit on antihelium is: $\mathrm{N}($ antihelium $) / \mathrm{N}($ helium $)<1.1 \cdot 10^{-6}$ over the range 1 to $140 \mathrm{GV}$ in rigidity. The same study 

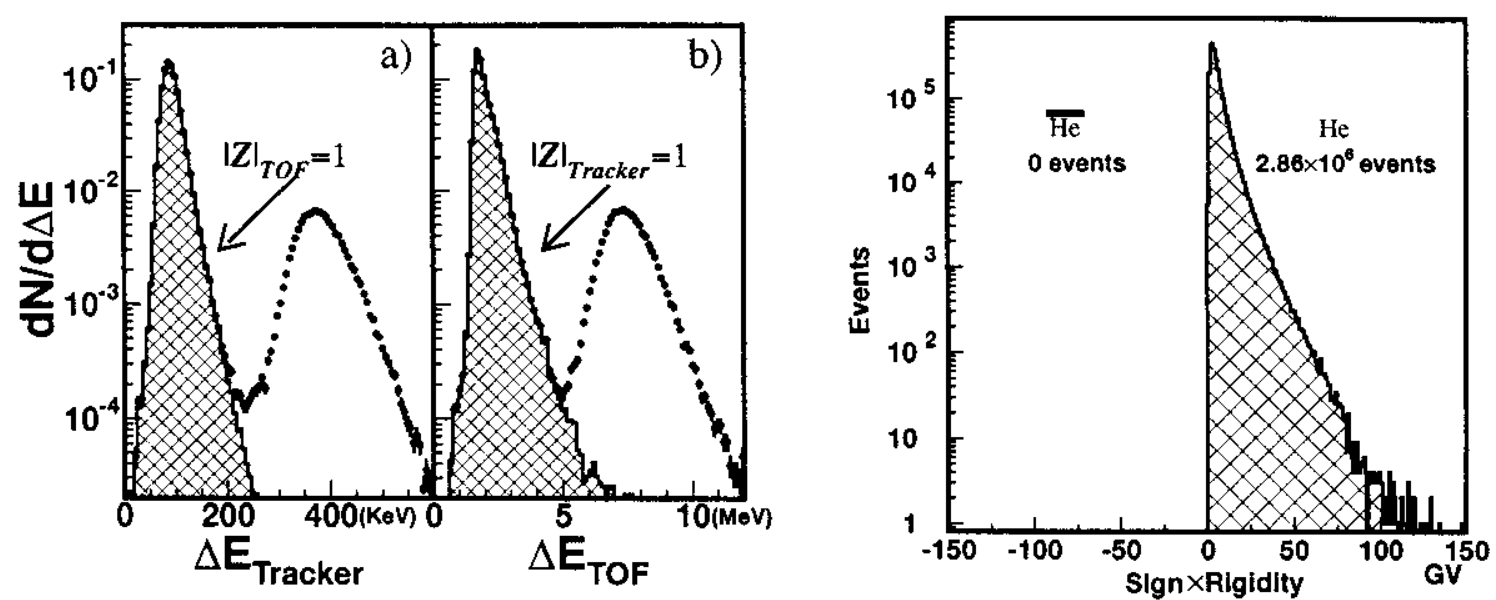

Figure 2: Charge separation from $\mathrm{dE} / \mathrm{dx}$ (left) and Measured rigidity times the charge sign for selected $|Z|=2$ events (right)

applied to nuclei with $|Z|>2$ gave $1.56 \cdot 10^{5}$ events in the same rigidity range and no antinucleus candidate.

\section{Measurement of spectra at high energy}

Spectra of cosmic rays are generally expected to fall off like a power law at high energy. A measurement of the slope for protons and helium nuclei has been done with the AMS-01 data ${ }^{13,14}$.

Protons and Helium are easy to study since they are the most abundant charged cosmic rays. All the other ones are orders of magnitude lower in flux. The data used for this study were restricted to the periods when the axis of AMS was pointing toward the Zenith within $45^{\circ}$. It was required to have at least 4 hits in the tracker in the bending plane and 3 in the non-bending plane in order to fit the particle rigidity $\mathrm{R}=p c /|Z| \cdot e$. The charge was measured from energy loss in tracker and TOF, leaving less than $10^{-4}$ proton contamination in the Helium events whose flux is typically 10 times lower. The remaining background events in the proton sample from pions produced in the top part of AMS was found to be less than $0.5 \%$ below $1 \mathrm{GeV}$ and vanishing rapidly with energy, while the contamination of deuterons was reduced to a negligible level by requiring the measured mass to be within 3 standard deviations of the proton mass. The distributions obtained were corrected for the differential acceptance of the detector as a function of the latitude and of the pointing angle, giving a contribution to the total systematic error of $5 \%$. They were then unfolded for the effect of the rigidity resolution. After applying all these corrections the spectrum was parametrized by a power law in rigidity as:

$$
\Phi_{0} \cdot R^{-\gamma}
$$

Fitting the parameters on the rigidity range 10 to $200 \mathrm{GV}$ for the protons yields :

$$
\begin{gathered}
\gamma=2.78 \pm 0.009(\text { fit }) \pm 0.019(\text { sys }) \\
\Phi_{0}=[17.1 \pm 0.15(\text { fit }) \pm 1.3(\text { sys }) \pm 1.5(\gamma)] G V^{2.78} /\left(m^{2} . \text { sec.sr. } M V\right)
\end{gathered}
$$

Fitting the parameters on the rigidity range 20 to $200 \mathrm{GV}$ for the Helium yields:

$$
\begin{gathered}
\gamma=2.740 \pm 0.010(\text { fit }) \pm 0.016(\text { sys }) \\
\Phi_{0}=[2.52 \pm 0.09(\text { fit }) \pm .13(\text { sys }) \pm .14(\gamma)] G V^{2.74} /\left(m^{2} . \text { sec.sr. } M V\right)
\end{gathered}
$$




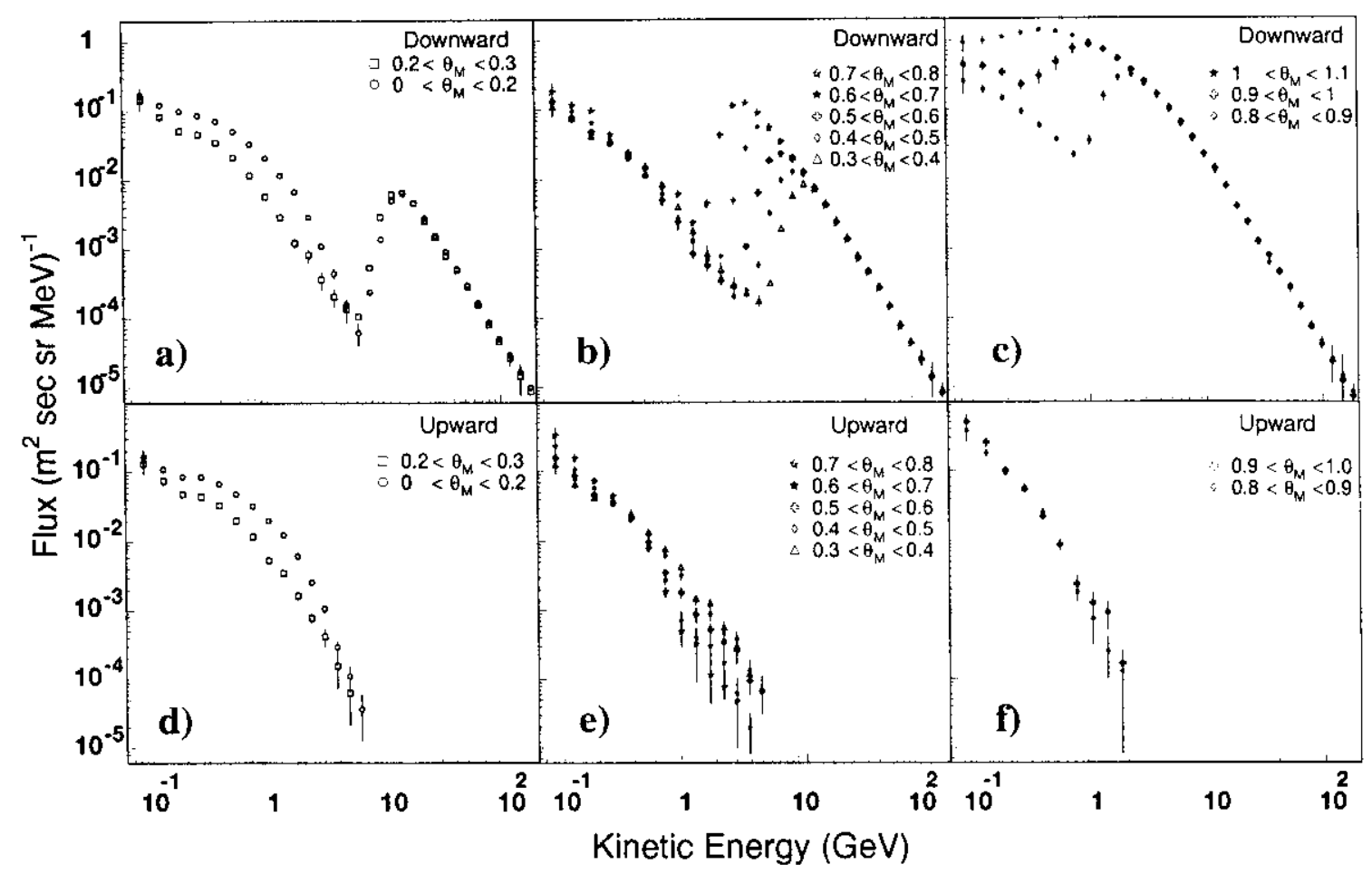

Figure 3: Fluxes of proton upward and downward in bins of geomagnetic latitude

\section{Particles spectra near and below geomagnetic threshold}

\subsection{Protons fluxes}

Due to the difficulty to launch and operate a magnetic spectrometer in space, the previous measurements of charged particles at high energy were done with balloon-borne experiment, flying at a maximum altitude of $40 \mathrm{kms}$, where there is still $5 \mathrm{~g} / \mathrm{cm}^{2}$ of atmosphere above. It had been demonstrated by Stoermer ${ }^{8}$ ] that if there was under cutoff particles they could not come from the outer space, but rather from direct interaction in the atmosphere. Under cutoff particles were detected and classified in 3 categories : i) Atmospheric Secondaries, for the particles produced in the air above the detector and going downward ii) Splash Albedo for upward going particles produced by interaction in the air iii) Return Albedo for the fraction of splash albedo going back to the earth on the opposite atmosphere. Particle produced and then detected in these experiments were immediately absorbed in the atmosphere. At higher altitude like the $400 \mathrm{~km}$ orbit of AMS, no measurement existed above $200 \mathrm{MeV}$.

It was a surprise in AMS to observe a large flux of under cutoff particles up to few $\mathrm{GeV}$ in energy, and that around the magnetic equator particles were trapped for a long duration ${ }^{11}$.

For the study of the proton flux, the sample was restricted to the period in which the z-axis was pointing within $1^{\circ}$ of the zenith (downward going particles) or of the Nadir (upward going particles). The background was found negligible like at higher energy and the corrections for the differential acceptance and the detector resolution were the same. The effect of geomagnetic cutoff shows up very neatly as a function of the geomagnetic latitude. At high energy, say above $10 \mathrm{GeV}$, all the spectra have the same behavior. Surprisingly, a second (secondary) spectrum starts below the geomagnetic cutoff with a rate about an order of magnitude below. Near the equator the secondary spectrum has a distinct structure. In the region $\Theta_{M}<0.3$, the spectrum extend from the lowest measured energy, $0.1 \mathrm{GeV}$ to about $6 \mathrm{GeV}$, with a rate of $70 \mathrm{~m}^{-2} \cdot \mathrm{sec}^{-1} \cdot \mathrm{sr}^{-1}$. In all the bins in latitude up to $\Theta_{M}=0.7$ the secondary spectrum of downward particle has a flux equal to the one of upward particles measured with the detector 


\section{Particles detected at $\Theta M<0.3$ rad}

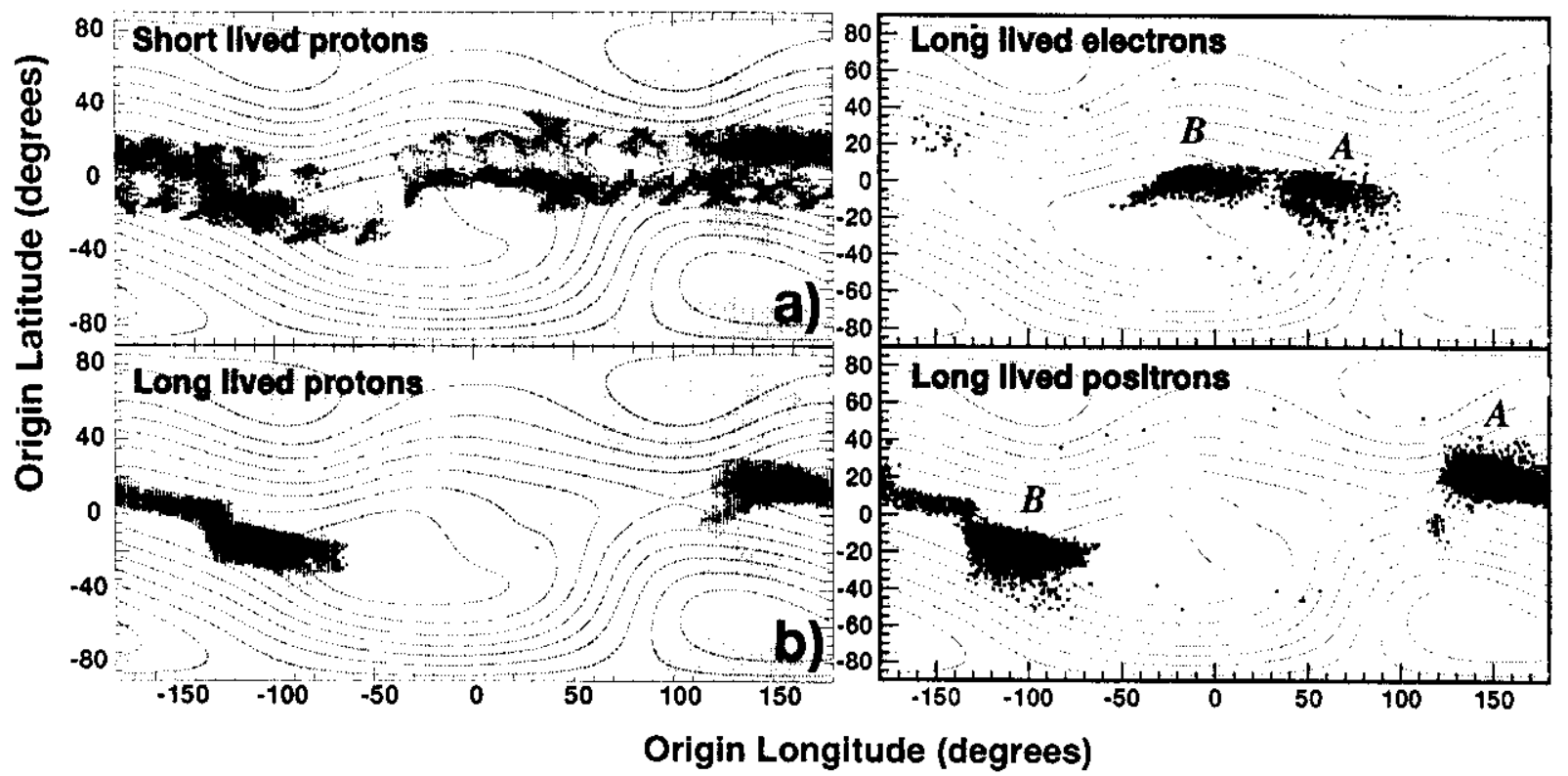

Figure 4: Geographical origin of a) short-lived and b) long-lived protons with $p<3 G e V / c$. The dashed lines indicate geomagnetic field contour at $380 \mathrm{~km}$.

pointing toward the earth (fig 5). By tracing back $10^{5}$ protons of the secondary flux in the earth magnetic field and stopping the extrapolation either when the particle reach the top of the atmosphere defined at $40 \mathrm{kms}$, or when the flight time reached 10 seconds, it was found that all the protons from the secondany spectrum originate in the atmosphere. $30 \%$ of the protons flew for less than 0.3 seconds (so-called "short lived" particles) and their origin in the atmosphere is uniformly distributed around the globe reflecting the shuttle orbits. The remaining $70 \%$, socalled long-lived particles, flew for more than $0.3 \mathrm{sec}$ and originate from a restricted geographic region (Fig. 4). This global behaviour with a complicated path of particles in the geomagnetic field is different from what is observed either with balloon-borne experiments in which particles are all "short lived", or with satellites in the radiation belts where the protons bounce across the equator for a much longer time.

\subsection{Electron and Positron measurements}

AMS was also able to measure electrons and positrons ${ }^{12}$. The electrons thanks to their negative sign have clear identification and very small background, hence AMS was able to measure their spectra with limited statistics up to energies of $100 \mathrm{GeV}$. For a geomagnetic latitude of $\Theta_{M}<0.3$, it was found that the electron spectrum has the same behaviour as the proton one, with a secondary flux below the geomagnetic cutoff extending up to $3 \mathrm{GeV}$ (fig 5) and the same amount of electrons moving downward and upward, uniformly spread around the globe. For positrons, the high flux of protons with a rate about thousand time higher gives a lot of background at high energy, hence the study was done only below $2 \mathrm{GeV}$, an energy range where the time of flight of the particle and the signal in the aerogel counter allows to reduce the proton misidentification to a negligible level. As a consistency check, the positron fraction over the sum of positron and electron rate was compared in the polar region $\left(\Theta_{M}>1.0\right)$ to the previous measurement by balloon borne experiments and was found in excellent agreement.

The electron and positron spectra exhibits the same behaviour as protons spectra, with a secondary flux below the geomagnetic threshold which contains an equal number of downward and upward going particles. In the geomagnetic equator region $\Theta_{M}<0.3$ radian the energy range 

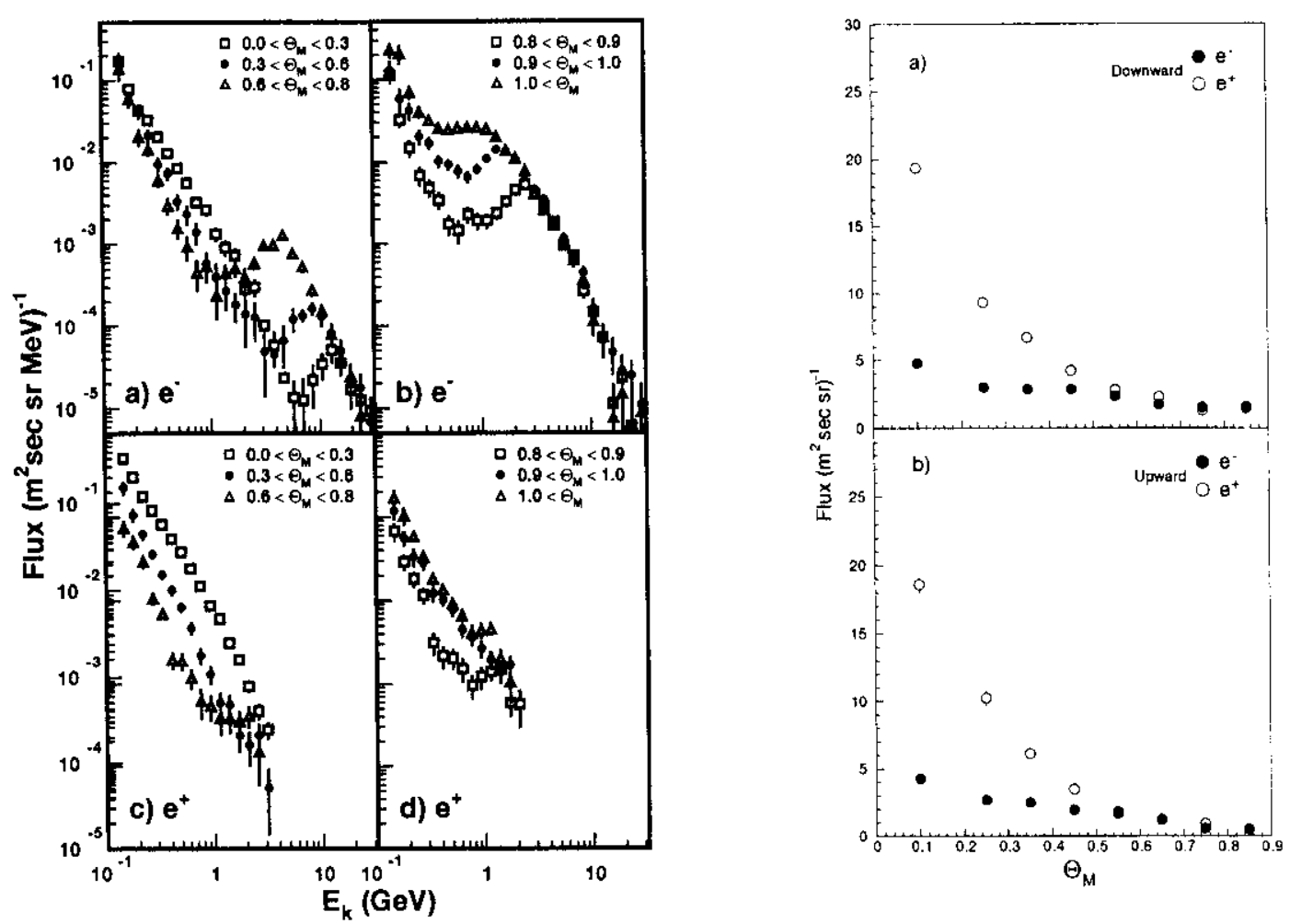

Figure 5: Electrons and positrons as a function of geomagnetic latitude. On the left the flux spectra for downward going electrons $(\mathrm{a}, \mathrm{b})$ and positrons $(\mathrm{c}, \mathrm{d})$ are shown. On the right the propoerties of second lepton spectra flux :

(a) downward and (b) upward going electrons and positrons integrated over the range $0.2-2.5 \mathrm{GeV}$

0.1 to $2.5 \mathrm{GeV}$, the sample contains also the so-called short-lived and long-lived particles. Shortlived electrons and positrons origin reflects the orbits of the shuttle while long-lived positrons come from the same limited geographical region than protons. Long-lived electrons originate from a restricted geographical region which is complementary of the one of protons and electrons. Eventually, the flux of positrons becomes 5 times higher than the electron one in the equator region.

\subsection{Measurement of Helium fluxes and mass spectrum}

Below the energy threshold after the fall of the primary spectrum a secondary spectrum shows up, 2 to 3 order of magnitude smaller in flux, in the equatorial region $\left|\Theta_{M}\right|<0.4$ rad. Fitting the mass of helium nuclei above the threshold one gets $M=3.64 \pm 0.01 \mathrm{GeV} / \mathrm{c}^{2}$ in the polar region $\left|\Theta_{M}\right|>0.4 \mathrm{rad}$, and $\mathrm{M}=3.65 \pm 0.09 \mathrm{GeV} / \mathrm{c}^{2}$ in the equatorial region $\left|\Theta_{M}\right|<0.4 \mathrm{rad}$. Comparing with the $\mathrm{He}^{4}$ mass of $3.727 \mathrm{GeV} / \mathrm{c}^{2}$, it shows that the content is mostly $\mathrm{He}^{4}$. However, the same mass fitting applied to Helium nuclei below the threshold in the equatorial region gives a mass of $2.86 \pm 0.04 \mathrm{GeV}$ (fig 6), close to the $H e^{3}$ mass of 2.809 $\mathrm{GeV} / \mathrm{c}^{2}$ which implies that these nuclei are mostly $\mathrm{He}^{3}$ which could be due to spallation of $\mathrm{He}^{4}$ on the atmosphere.

\section{Conclusion on physics results of AMS01.}

The spectra of protons, electrons, positrons, and Helium in cosmic rays exhibit all the same behaviour, with a secondary flux below the geomagnetic threshold a a concentration of particle trapped around the equator. From the simulation of the trajectory of high energy cosmic rays in the earth magnetic field, it appears that these particles trapped at high altitude in the magnetic 

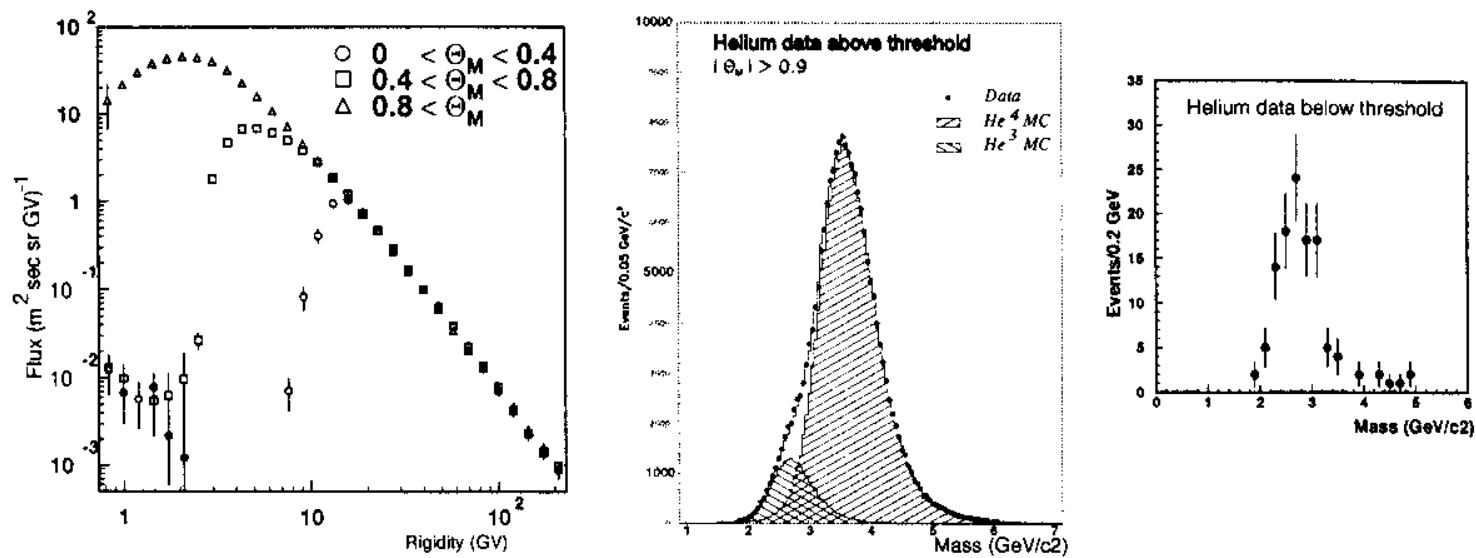

Figure 6: The left part of figure shows the spectra of Helium nuclei in bins of geomagnetic latitude, which exhibits also a secondary spectrum. The central figure shows the mass distribution of Helium candidates above threshold, compatible with a strong content of Helium 4 , while the right figure shows the mass distribution of particles below threshold which is compatible with Helium 3.

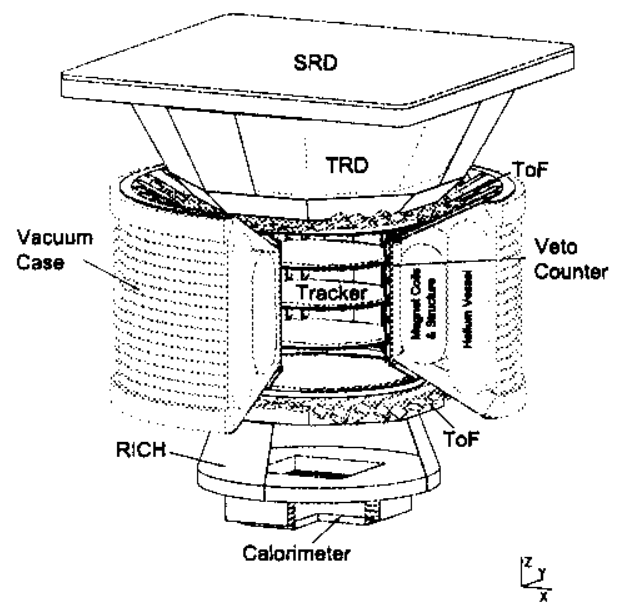

Figure 7: The AMS02 detector for the ISS

field are secondaries of the interaction of primary cosmic rays with the atmosphere, forming a kind of belt. The excess of positrons near the equator is also explained by the simulation. Finally. it must be remarked that, though the flight STS-91 was only a technological test, it gave numerous, precise, and sometimes unexpected results on charged cosmic rays, which demonstrates the quality of the instrument ${ }^{15}$.

\section{AMS on the international space station ISS}

AMS is scheduled to fly to the international space station ISS in October 2003, and to take data for 3 to 5 years. In order to fulfill the program of physics, specially for the high energy part of the cosmic rays up to a few $\mathrm{TeV}$, the instrument will be modified and completed with new detectors (fig 7) to both improve measurements accuracies and particles identification.

The permanent magnet will be replaced by a superconducting one with the same inner volume, the same configuration of field, and a bending power 6 times higher $B L^{2}=0.86 T m^{2}$. This will allow to measure particle momenta with a resolution better than $2 \%$ up to $100 \mathrm{GeV}$ and to have a excellent determination of the sign of electric charge up to several $\mathrm{TeV}$. The tracker will be upgraded from 6 to 8 double-sided planes of silicon detectors. A Transition Radiation Detector placed above the TOF detector, made of 20 planes of straw tubes and foam, 
will give a separation power of nearly 200 between hadrons and electrons up to $500 \mathrm{GeV}$, and will give additional points on the trajectory of particles. Below the magnet, a RICH cerenkov counter with a double radiator of aerogel and Sodium fluoride will allow to identify nuclei up to Aluminium, and to disentangle the isotopic content up to Oxygen for nuclei with an energy per nucleon below $12 \mathrm{GeV}$. Underneath the $\mathrm{RICH}$, an imaging electromagnetic calorimeter made of a sandwich of lead and scintillating fibers of depth $16.5 X_{0}$ will measure the electromagnetic shower energy with an accuracy of a few percent. Thanks to its very fine granularity (18 sampling in depth, and a transverse granularity of 0.5 Mollier radius in both direction) the calorimeter will allow to achieve a rejection power hadrons/electron close to $10^{4}$, which combined with the TRD will achieve the identification power necessary for the study of the charged cosmic rays up to the TeV range. Eventually, a Synchrotron Radiation Detector placed above the TRD will detect the synchrotron radiation produced by electrons/positrons of energy above $500 \mathrm{GeV}$ in the earth magnetic field before traversing the detector and will allow to increase the range of energy covered by AMS for electrons and positrons to $3 \mathrm{TeV} .20000$ such particles are expected between $500 \mathrm{GeV}$ and $3 \mathrm{TeV}$ during the 3 years of data taking.

\section{Conclusion}

The AMS02 experiment on the International Space Station will open a domain of physics yet largely unexplored. The precursor flight dedicated to test the technology and the conditions in space gave numerous original and unexpected physics results, which demonstrates the capability of the detector to fulfill its physics program.

\section{Acknowledgments}

I am very indebted to Professor Samuel C.C. Ting for giving me the opportunity to present the results of AMS at this conference. I would like also to thank all my colleagues of the AMS experiment and specially the AMS group at LAPP for many fruitful discussions. The financial support of the IN2P3/CNRS, the "Conseil Regional Rhone-Alpes", and the "Conseil General de Haute-Savoie" is gratefully acknowledged.

\section{References}

1. A. D. Sacharov, JETP Lett. 5, 24, (1967).

2. R.L. Golden et al, $A p J 479,992,(1997)$.

3. J.F.Ormes et al., ApJ Letters 482, L187, (1997).

4. T. Saeki et al., Phys. Lett. B 422, 319, (1998).

5. V.S. Berezinsky et al., in TAUP 95 Conference, Nucl. Phys. B, Proc. Suppl. : 48 (1996)

6. E. Diehl et al., Phys.Rev. D 52, 7 (1995).

7. F. Barao et al, The AMS-01 Aerogel Threshold Cerenkov counter Submitted to Nucl. Instrum. Methods A.

8. C. Störmer, The Polar Aurora, (Clarendon Press) Cambridge 1955.

9. See C. Cecchi, this Conference. J. Favier et al, To be published. L. Derome et al, To be published

10. J. Alcazar et al., AMS Collaboration, Phys. Lett. B461, 387 (1999).

11. J. Alcazar et al., AMS Collaboration,Phys. Lett. B472, 215 (2000).

12. J. Alcazar et al., AMS Collaboration,Phys. Lett. B484, $10(2000)$.

13. J. Alcazar et al., AMS Collaboration,Phys. Lett. B490, 27 (2000).

14. J. Alcazar et al., AMS Collaboration, Submitted to Phys. Lett. B.

15. J. Alcazar et al., AMS Collaboration, Physics Report in preparation. 